\title{
Thérapie génique et immunothérapie active du cancer
}

Les essais d'immunothérapie du cancer sont extrêmement anciens ; ils sont fondés sur l'expression, par la cellule cancéreuse, de néo-antigènes contre lesquels une réponse immune de type cytotoxique pourrait être développée et avoir un effet protecteur [1, 2]. Cette réponse est cependant le plus souvent trop faible pour permettre d'obtenir un réel effet anti-tumoral. C'est la raison pour laquelle, les techniques de transfert de gènes et de thérapie génique se développant, plusicurs équipes ont décidé de tenter d'augmenter la réponse immune en agissant, soit sur l'expression d'un néo-antigène immunisant, soit sur celles de lymphokines stimulatrices de l'immunité. Dans la première catégoric, on peut ranger des essais en cours dans l'équipe de Rosenberg (NIH, Bethesda, MD, USA) consistant à faire exprimer par des cellules de grandes quantités de néo-antigènes de mélanome. Dans la seconde, on trouve l'utilisation de cellules hyper-exprimant diverses cytokines, le TNF (tumor necrosis factor) $(\mathrm{m} / \mathrm{s}$ $n^{\circ} 2$, vol. 6, p. 144 et $n^{\circ} 1$, vol. 7, p. 83), l'interleukine 2 [3] et, plus récemment, l'interlcukine 4 . La sécrétion par les cellules tumorales de grandes quantités de ces interleukines pourrait stimuler le développement de cellules $\mathrm{T} \mathrm{CD}^{+}$cytotoxiques reconnaissant des néo-antigènes présentés par des molécules de classe I du complexe majeur d'histocompatibilité. Une équipe de Baltimore, dans le Maryland (USA) [4], vient de montrer l'efficacité de cette stratégie en utilisant l'interleukine 4 (IL-4) produite par les cellules de carcinome rénal de souris. IL-4 est un facteur de croissance ou de différenciation des lymphocytes $\mathrm{B}$ et $\mathrm{T}$. Il semble augmenter le potentiel cytolytique des lymphocytes $\mathrm{T}$ cytotoxiques.

caine est celui d'un carcinome spontané de la souris mimant le cancer du rein chez l'homme. Les cellules tumorales ont été prélevées, puis transfectées avec l'ADN recombinant d'un vecteur polyome contenant un $\mathrm{ADNc}$ codant pour IL-4. Lorsque ces cellules sont réinjectées à une souris immunocompétente $\mathrm{Balb} / \mathrm{c}$, elles sont rejetées totalement, même dans le cas où l'inoculum est très important. Ce rejet exige la présence de lvmphocytes $\mathrm{T}$ car des souris immunodéprimées (souris nude) ou SCID (severe combined immunodeficieng) finissent par développer des tumeurs. Le rejet tumoral est associé à un infiltrat inflammatoire comportant des cellules macrophagiques activées. L'immunisation préalable par des cellules cancéreuses produisant de l'IL-4 recombiné confère aussi une bonne immunité contre des cellules parentales non modifiées injectées en un autre site. Cet effet exige la présence de lymphocytes $\mathrm{CD}^{+}$alors que les cellules auxiliaires $\mathrm{CD}^{+}{ }^{+}$semblent jouer un rôle plus modeste. L'effet antitumoral est spécifique des antigènes de la cellule cancéreuse puisque l'immunité acquise contre un type de tumeurs ne vaut pas contre un autre. Cependant, lorsque deux types de cellules tumorales différentes, l'une hyperproduisant de l'IL-4, l'autre non, sont coinjectées en un même site, une immunité apparaît contre toutes deux. En conclusion, la présence de cellules néoplasiques synthétisant de larges quantités d'IL-4 induit une immunisation spécifique qui exige la présence de lymphocytes $\mathrm{CD}^{+}$cytotoxiques et s'accompagne d'une forte induction des cellules présentatrices d'antigènes que sont les macrophages. Ce dernier point différencie les essais utilisant des cellules produisant de l'IL-4 de ceux faisant appel à l'IL-2. Dans ce dernier cas, en effet, les tumeurs en voie de rejet sont avant tout infiltrées par des cellules $\mathrm{T}$ cytotoxiques. Chez l'homme, un effet similaire pourrait être espéré de l'utilisation de lymphocytes recombinés à la condition expresse que ceuxci se localisent électivement au niveau du tissu tumoral où ils doivent s'immuniser. L'utilisation des TIL (tumor infiltrating lymphocytes) n'est pas une garantie absolue de ciblage correct. Alternativement, une immunothérapie à l'aide de cellules tumorales cultivées ex vivo, transduites à l'aide d'un rétrovirus recombiné et réinjectées pourrait, dans l'avenir, s'envisager dès lors que l'efficacité de cette entreprise et, surtout, sa non-dangerosité, auront été vérifiées par des travaux expérimentaux suffisants.

A.K.

1. Max J. Cancer vaccines show promise at last. Science 1989 ; 245 : 813-5.

2. Lurquin C, Van Pel A, Mariamé B, et al. Structure of the gene of Tum - transplantation antigen P91A : the mutated exon encodes a peptide recognized with $\mathrm{L}^{\mathbf{d}}$ by cytolytic $\mathbf{T}$ cells. Cell 1989 ; 58 : 293-303.

3. Fearon ER, Pardoll DM, Itaya $\mathrm{T}$, et al. Interleukin 2 production by tumor cells bypasses $T$ helper function in the generation of an antitumor response. Cell 1990; 60 : 397-403. 4. Golumbek PT, Lazenby AJ, Levitsky HI, et al. Treatment of established renal cancer by tumor cells engineered to secrete interleukine 4 . Science 1991; 254: 713-6. 